\title{
Poly-central factorial sequences and poly-central-Bell polynomials
}

\author{
Hye Kyung Kim ${ }^{1 *}$ (D) and Taekyun Kim²
}

"Correspondence: hkkim@cu.ac.kr 'Department of Mathematics Education, Daegu Catholic University, Gyeongsan 38430, Republic of Korea Full list of author information is available at the end of the article

\section{是 Springer}

\begin{abstract}
In this paper, we introduce poly-central factorial sequences and poly-central Bell polynomials arising from the polyexponential functions, reducing them to central factorials and central Bell polynomials of the second kind respectively when $k=1$. We also show some relations: between poly-central factorial sequences and power of $x$; between poly-central Bell polynomials and power of $x$; between poly-central Bell polynomials and the poly-Bell polynomials; between poly-central Bell polynomials and higher order type 2 Bernoulli polynomials of second kind; recurrence formula of poly-central Bell polynomials.

MSC: $11 \mathrm{~B} 73 ; 11 \mathrm{~B} 83 ; 05 \mathrm{~A} 19$

Keywords: Central factorials; Central-Bell polynomials and numbers; Modified polyexponential functions; Stirling numbers of the first and second kind; Type 2 Bernoulli polynomials of the second kind
\end{abstract}

\section{Introduction}

The central factorial numbers of the first and second kinds consist of the same kind of reciprocity as the corresponding polynomials for the Stirling numbers of the first and second kinds [20]. They have appeared in many different contexts as follows: the approximation theory [2], algebraic geometry [6, 20], and spectral theory of differential operators [7, 18]. The poly-exponential functions were reconsidered by Kim [9] in view of an inverse to the polylogarithm functions which were first studied by Hardy [8]. Kim et al. showed that the degenerate Daehee numbers of order $k$ expressed the degenerate polyexponential functions in [13]. Furthermore, recently, Kim and Kim introduced the poly-Bell polynomials and the poly-Lah-Bell polynomials arising from polyexponential functions respectively in $[16,17]$. With this in mind, we introduce poly-central factorial sequences and poly-central Bell polynomials arising from the polyexponential functions, reducing them to central factorials and central Bell polynomials of the second kind respectively when $k=1$. We also show some relations: between poly-central factorial sequences and power of $x$; between poly-central Bell polynomials and power of $x$; between poly-central Bell polynomials and the poly-Bell polynomials; between poly-central Bell polynomials and higher order type 2 Bernoulli polynomials of second kind; recurrence formula of poly-central Bell polynomials.

(c) The Author(s) 2021. This article is licensed under a Creative Commons Attribution 4.0 International License, which permits use sharing, adaptation, distribution and reproduction in any medium or format, as long as you give appropriate credit to the original author(s) and the source, provide a link to the Creative Commons licence, and indicate if changes were made. The images or other third party material in this article are included in the article's Creative Commons licence, unless indicated otherwise in a credit line to the material. If material is not included in the article's Creative Commons licence and your intended use is not permitted by statutory regulation or exceeds the permitted use, you will need to obtain permission directly from the copyright holder. To view a copy of this licence, visit http://creativecommons.org/licenses/by/4.0/. 
First, definitions and preliminary properties required in this paper are introduced.

The central factorial $x^{[n]}$ is defined by

$$
\begin{aligned}
x^{[0]} & =1, \\
x^{[n]} & \left.=x\left(x+\frac{n}{2}-1\right)\left(x+\frac{n}{2}-2\right)\right) \cdots\left(x-\frac{n}{2}+1\right), \quad(n \geq 1),(\text { see }[1,3,10,11,19]) .
\end{aligned}
$$

The central factorial $x^{[n]}$ is given by the generating function

$$
\sum_{n=0}^{\infty} x^{[n]} \frac{t^{n}}{n !}=\left(\frac{t}{2}+\sqrt{1+\frac{t^{2}}{4}}\right)^{2 x} \quad(\text { see }[10,11,19]) .
$$

For any nonnegative integer $n$, the central factorial numbers of the first kind are given by

$$
x^{[n]}=\sum_{l=0}^{n} t(n, l) x^{l} \quad(\text { see }[20]) .
$$

By (3), we easily get

$$
\frac{1}{l !}\left(2 \log \left(\frac{t}{2}+\sqrt{1+\frac{t^{2}}{4}}\right)\right)^{l}=\sum_{n=l}^{\infty} t(n, l) \frac{t^{n}}{n !} \quad(\text { see }[10,11,19])
$$

where $t \in \mathbb{C}$ with $|t|<1$.

$$
\begin{aligned}
\text { Let } f(t) & =2 \log \left(\frac{t}{2}+\sqrt{1+\frac{t^{2}}{4}}\right) \text {. Then } \\
f^{-1}(t) & =e^{\frac{t}{2}}-e^{-\frac{t}{2}}
\end{aligned}
$$

which is the compositional inverse of the function $f(t)$.

From (4) and (5), the central factorial numbers of the second kind are given by

$$
\frac{1}{l !}\left(e^{\frac{t}{2}}-e^{-\frac{t}{2}}\right)^{l}=\sum_{n=l}^{\infty} T(n, l) \frac{t^{n}}{n !} \quad(\text { see }[10,11,19])
$$

Riordan showed that the central factorial numbers of the second kind $T(n, k)$ are the coefficients in the expansion of $x^{n}$ in terms of central factorials given by

$$
x^{n}=\sum_{l=0}^{n} T(n, l) x^{[l]} \quad(n \geq l \geq 0),(\text { see }[19]) .
$$

Kim and Kim introduced the central Bell polynomials $B_{n}^{(c)}(x)$ defined by

$$
B_{n}^{(c)}(x)=\sum_{k=0}^{n} x^{k} T(n, k), \quad(n \geq 0),(\text { see }[11])
$$


and the central Bell numbers $B_{n}^{(c)}$ by $B_{n}^{(c)}(1)$, so that

$$
B_{n}^{(c)}=\sum_{k=0}^{n} T(n, k), \quad(n \geq 0)
$$

From (6) and (8), we note that the generating function for the central Bell polynomials is

$$
e^{x\left(e^{\frac{t}{2}}-e^{-\frac{t}{2}}\right)}=\sum_{n=0}^{\infty} B_{n}^{(c)}(x) \frac{t^{n}}{n !} \quad(\text { see }[11])
$$

where $B_{n}^{(c)}(x)=\sum_{k=0}^{n} T(n, k) x^{k}$ are the central polynomials Bell polynomials and $B_{n}^{(c)}=$ $B_{n}^{(c)}(1)$ are the central Bell numbers.

For $n \geq 0$, the Stirling numbers of the first kind $S_{1}(n, l)$ are the coefficients of $x^{l}$ in

$$
(x)_{n}=\sum_{l=0}^{n} S_{1}(n, l) x^{l} \quad(\text { see }[3,5,15]) .
$$

From (10), it is easy to see that

$$
\frac{1}{k !}(\log (1+t))^{k}=\sum_{n=k}^{\infty} S_{1}(n, k) \frac{t^{n}}{n !} .
$$

In the inverse expression to (10), for $n \geq 0$, the $n$th power of $x$ can be expressed in terms of the Stirling numbers of the second kind $S_{2}(n, l)$ as follows:

$$
x^{n}=\sum_{l=0}^{n} S_{2}(n, l)(x)_{l} \quad(\text { see }[3,5,15]) .
$$

From (12), it is easy to see that

$$
\frac{1}{k !}\left(e^{t}-1\right)^{k}=\sum_{n=k}^{\infty} S_{2}(n, k) \frac{t^{n}}{n !} .
$$

The $n$th Bell number $B_{n}(n \geq 0)$ is the number of ways to partition a set with $n$ elements into nonempty subsets. The Bell polynomials are natural extensions of the Bell numbers as follows:

$$
b e l_{n}(x)=\sum_{k=0}^{n} S_{2}(n, k) x^{n} .
$$

It is well known that the generating function of the Bell polynomials is given by

$$
e^{x\left(e^{t}-1\right)}=\sum_{n=0}^{\infty} b e l_{n}(x) \frac{t^{n}}{n !} \quad(\text { see }[3,5,16]) .
$$

Now, as well established within academia, the ordinary Bernoulli polynomials $b_{n}(x)$ and the Euler polynomials $E_{n}(x),(n \in \mathbb{N} \cup\{0\})$ are respectively defined by their generating 
functions as follows (see $[3,5])$ :

$$
\left(\frac{t}{e^{t}-1}\right) e^{x t}=\sum_{n=0}^{\infty} b_{n}(x) \frac{t^{n}}{n !}, \quad \frac{2}{e^{t}+1} e^{x t}=\sum_{n=0}^{\infty} E_{n}(x) \frac{t^{n}}{n !} .
$$

When $x=0, b_{n}=b_{n}(0)$ and $E_{n}=E_{n}(0)$ are respectively called the Bernoulli numbers and the Euler numbers.

For $r \in \mathbb{R}$, the type 2 Bernoulli polynomials of the second kind with order $r$ are defined by the generating function

$$
\left(\frac{(1+t)-(1+t)^{-1}}{2 \log (1+t)}\right)^{r}(1+t)^{x}=\sum_{n=0}^{\infty} b_{n}^{*(r)}(x) \frac{t^{n}}{n !} \quad \text { (see [12]). }
$$

When $x=0, b_{n}^{*(r)}(0)$ are called the type 2 Bernoulli numbers of the second kind order $r$.

Kim and Kim introduced the modified polyexponential function as

$$
J_{k}(x)=\sum_{n=1}^{\infty} \frac{x^{n}}{(n-1) ! n^{k}} \quad(k \in \mathbb{Z}),(\text { see }[9,14]) .
$$

When $k=1$, we see that $\operatorname{Ei}_{1}(x)=e^{x}-1$.

Recently, the poly-Bell polynomials were introduced by

$$
1+J_{k}\left(x\left(e^{t}-1\right)\right)=\sum_{n=0}^{\infty} b e l_{n}^{(k)}(x) \frac{t^{n}}{n !} \quad(\text { see }[16])
$$

and $b e l_{0}^{(k)}(x)=1$.

When $k=1$, from (15), we note that

$$
\begin{aligned}
1+J_{1}\left(x\left(e^{t}-1\right)\right) & =1+\sum_{n=1}^{\infty} \frac{\left(x\left(e^{t}-1\right)\right)^{n}}{(n-1) ! n} \\
& =\exp \left(x\left(e^{t}-1\right)\right)=\sum_{n=0}^{\infty} b e l_{n}(x) \frac{t^{n}}{n !} .
\end{aligned}
$$

From (20), we have

$$
b e l_{n}^{(1)}(x)=\operatorname{bel}_{n}(x)
$$

\section{Poly-central factorial sequences and poly-central-Bell polynomials}

In this section, we define poly-central factorial sequences and poly-central-Bell polynomials respectively by using the degenerate polylogarithm functions and give explicit expressions and recurrence formula of poly-central Bell polynomials.

First, we consider the poly-central factorial sequences $x^{[n](k)}$, which are derived from the polyexponential function to be

$$
1+J_{k}\left(2 x \log \left(\frac{t}{2}+\sqrt{1+\frac{t^{2}}{4}}\right)\right)=\sum_{n=0}^{\infty} x^{[n](k)} \frac{t^{n}}{n !} \quad \text { and } \quad x^{[0](k)}=1 .
$$


When $k=1$, since $J_{1}(x)=e^{x}-1$, we note that

$$
1+J_{1}\left(2 x \log \left(\frac{t}{2}+\sqrt{1+\frac{t^{2}}{4}}\right)\right)=\left(\frac{t}{2}+\sqrt{1+\frac{t^{2}}{4}}\right)^{2 x}=\sum_{n=0}^{\infty} x^{[n]} \frac{t^{n}}{n !} .
$$

Therefore, by (22), we have $x^{[n](1)}=x^{[n]}$.

Second, we define the poly-central-Bell polynomials $B_{n}^{(c, k)}(x)$, which arise from the polyexponential function to be

$$
1+J_{k}\left(x\left(e^{\frac{t}{2}}-e^{-\frac{t}{2}}\right)\right)=\sum_{n=0}^{\infty} B_{n}^{(c, k)}(x) \frac{t^{n}}{n !} \quad \text { and } \quad B_{0}^{(c, k)}(x)=1 .
$$

When $k=1$, since $J_{1}(x)=e^{x}-1$, we note that

$$
1+J_{1}\left(x\left(e^{\frac{t}{2}}-e^{-\frac{t}{2}}\right)\right)=1+e^{x\left(e^{\frac{t}{2}}-e^{-\frac{t}{2}}\right)}-1=\sum_{n=0}^{\infty} B_{n}^{(c)}(x) \frac{t^{n}}{n !} .
$$

By (24), we have $B_{n}^{(c, 1)}(x)=B_{n}^{(c)}(x)$.

First, we observe relations of poly-falling factorial sequences and powers of $x$.

Theorem 1 For $k \in \mathbb{Z}$ and $n \geq 1$, we have

$$
x^{[n](k)}=\sum_{l=1}^{n} \frac{1}{l^{k-1}} t(n, l) x^{l}
$$

where $t(n, l)$ is the central factorial numbers of the first kind.

Proof By (4) and (21), we observe that

$$
\begin{aligned}
1+J_{k}\left(2 x \log \left(\frac{t}{2}+\sqrt{1+\frac{t^{2}}{4}}\right)\right) & =1+\sum_{l=1}^{\infty} \frac{(2 x)^{l}\left(\log \left(\frac{t}{2}+\sqrt{1+\frac{t^{2}}{4}}\right)\right)^{l}}{(l-1) ! l^{k}} \\
& =1+\sum_{l=1}^{\infty} \frac{x^{l}}{l^{k-1}} \frac{1}{l !}\left(2 \log \left(\frac{t}{2}+\sqrt{1+\frac{t^{2}}{4}}\right)\right)^{l} \\
& =1+\sum_{l=1}^{\infty} \frac{x^{l}}{l^{k-1}} \sum_{n=l}^{\infty} t(n, l) \frac{t^{n}}{n !} \\
& =1+\sum_{n=1}^{\infty}\left(\sum_{l=1}^{n} \frac{1}{l^{k-1}} t(n, l) x^{l}\right) \frac{t^{n}}{n !}
\end{aligned}
$$

Combining (21) with (25), we get the desired result.

In Theorem 1 , when $k=1$, we note that

$$
x^{[n]}=\sum_{l=0}^{n} t(n, l) x^{l}
$$


Theorem 2 For $k \in \mathbb{Z}$ and $n \geq 1$, we have

$$
x^{n}=n^{k-1} \sum_{l=0}^{n} T(n, l) x^{[l](k)}
$$

Proof By replacing $t$ with $e^{\frac{t}{2}}-e^{-\frac{t}{2}}$ in (21), from (18), the left-hand side of (21) is

$$
1+J_{k}(x t)=1+\sum_{n=1}^{\infty} \frac{x^{n} t^{n}}{(n-1) ! n^{k}}=1+\sum_{n=1}^{\infty} \frac{x^{n}}{n^{k-1}} \frac{t^{n}}{n !} .
$$

On the other hand, from (6), the right-hand side of (21) is

$$
\begin{aligned}
\sum_{m=0}^{\infty} x^{[m](k)} \frac{\left(e^{\frac{t}{2}}-e^{-\frac{t}{2}}\right)^{m}}{m !} & =\sum_{m=0}^{\infty} x^{[m](k)} \sum_{n=m}^{\infty} T(n, m) \frac{t^{n}}{n !} \\
& =\sum_{n=0}^{\infty} \sum_{m=0}^{n} x^{[m](k)} T(n, m) \frac{t^{n}}{n !}
\end{aligned}
$$

Comparing with the coefficients of (26) and (27), we have the desired result.

In Theorem 2, when $k=1$, we note that

$$
x^{n}=\sum_{l=0}^{n} t(n, l) x^{[l](k)}
$$

Theorem 3 For $k \in \mathbb{Z}$ and $n \geq 1$, we have

$$
B_{n}^{(c, k)}(x)=\sum_{l=1}^{n} \frac{1}{l^{k-1}} T(n, l) x^{l}
$$

Proof From (6) and (23), we observe that

$$
\begin{aligned}
1+J_{k}\left(x\left(e^{\frac{t}{2}}-e^{-\frac{t}{2}}\right)\right) & =1+\sum_{l=1}^{\infty} \frac{x^{l}\left(e^{\frac{t}{2}}-e^{-\frac{t}{2}}\right)}{(l-1) ! l^{k}} \\
& =1+\sum_{l=1}^{\infty} \frac{x^{l}}{l^{k-1}} \frac{\left(e^{\frac{t}{2}}-e^{-\frac{t}{2}}\right)^{l}}{l !} \\
& =1+\sum_{l=1}^{\infty} \frac{x^{l}}{l^{k-1}} \sum_{n=l}^{\infty} T(n, l) \frac{t^{n}}{n !} \\
& =1+\sum_{n=1}^{\infty}\left(\sum_{l=1}^{n} \frac{1}{l^{k-1}} T(n, l) x^{l}\right) \frac{t^{n}}{n !} .
\end{aligned}
$$

Combining (23) with (28), we get

$$
B_{n}^{(c, k)}(x)=\sum_{l=1}^{n} \frac{1}{l^{k-1}} T(n, l) x^{l} \quad(n \geq 1) .
$$


In Theorem 3 , when $k=1$, we note that

$$
B_{n}^{(c)}(x)=\sum_{l=0}^{n} T(n, l) x^{l}
$$

Theorem 4 For $k \in \mathbb{Z}$ and $n \geq 1$, we have

$$
B_{n}^{(c, k)}(x)=\sum_{m=1}^{n} \sum_{l=1}^{m}\left(\begin{array}{l}
n \\
m
\end{array}\right)\left(-\frac{1}{2}\right)^{n-m} l^{n-m-k+1} S_{2}(m, l) x^{l} .
$$

Proof From (13) and (23), we observe that

$$
\begin{aligned}
1+J_{k}\left(x\left(e^{\frac{t}{2}}-e^{-\frac{t}{2}}\right)\right) & =1+\sum_{l=1}^{\infty} \frac{x^{l} e^{-\frac{t}{2} l}\left(e^{t}-1\right)^{l}}{(l-1) ! l^{k}} \\
& =1+\sum_{l=1}^{\infty} \frac{x^{l} e^{-\frac{t}{2} l}}{l^{k-1}} \sum_{m=l}^{\infty} S_{2}(m, l) \frac{t^{m}}{m !} \\
& =1+\sum_{m=1}^{\infty} \sum_{l=1}^{m} \frac{1}{l^{k-1}} S_{2}(m, l) x^{l} \frac{t^{m}}{m !} \sum_{j=0}^{\infty}\left(-\frac{l}{2}\right)^{j} \frac{t^{j}}{j !} \\
& =1+\sum_{n=1}^{\infty}\left(\sum_{m=1}^{n} \sum_{l=1}^{m}\left(\begin{array}{c}
n \\
m
\end{array}\right) \frac{1}{l^{k-1}}\left(-\frac{1}{2} l\right)^{n-m} S_{2}(m, l) x^{l}\right) \frac{t^{n}}{n !} .
\end{aligned}
$$

Combining (23) with (29), we have the desired result.

In Theorem 4, when $k=1$, we note that

$$
B_{n}^{(c)}(x)=\sum_{m=1}^{n} \sum_{l=1}^{m}\left(\begin{array}{l}
n \\
m
\end{array}\right)\left(-\frac{1}{2} l\right)^{n-m} S_{2}(m, l) x^{l} .
$$

Theorem 5 For $k \in \mathbb{Z}$ and $n \geq 1$, we have

$$
B_{n}^{(c, k)}(x)=\sum_{l=1}^{n}\left(\begin{array}{l}
n \\
l
\end{array}\right)\left(-\frac{1}{2} l\right)^{n-l} b e l_{n-l}^{(k)}(x)
$$

where bel $_{n}^{(k)}(x)$ are the poly-Bell polynomials.

Proof From (19) and (23), we get

$$
\begin{aligned}
1+J_{k}\left(x\left(e^{\frac{t}{2}}-e^{-\frac{t}{2}}\right)\right) & =1+J_{k}\left(x e^{-\frac{t}{2}}\left(e^{t}-1\right)\right)=1+\sum_{l=1}^{\infty} \frac{x^{l} e^{-\frac{t}{2}}\left(e^{t}-1\right)^{l}}{(l-1) ! l^{k}} \\
& =1+\sum_{l=1}^{\infty} \frac{\left(x\left(e^{t}-1\right)\right)^{l}}{(l-1) ! l^{k}} \sum_{m=0}^{\infty}\left(-\frac{1}{2} l\right)^{m} \frac{t^{m}}{m !} \\
& =1+\sum_{l=1}^{\infty} b e l_{l}^{(k)}(x) \frac{t^{l}}{l !} \sum_{m=0}^{\infty}\left(-\frac{1}{2} l\right)^{m} \frac{t^{m}}{m !} \\
& =1+\sum_{n=1}^{\infty} \sum_{l=1}^{n}\left(\begin{array}{l}
n \\
l
\end{array}\right)\left(-\frac{1}{2} l\right)^{n-l} b e l_{n-l}^{(k)}(x) \frac{t^{n}}{n !} .
\end{aligned}
$$

Combining (23) with (30), we have the desired result. 
Theorem 6 For $k \in \mathbb{Z}$ and $n \geq 1$, we have

$$
\sum_{j=1}^{n} 2^{j} S_{1}(n, j) B_{j}^{(c, k)}(x)=\sum_{m=1}^{n} \sum_{l=1}^{m}\left(\begin{array}{c}
n \\
m
\end{array}\right) \frac{2^{l} x^{l}}{l^{k-1}} S_{1}(m, l) b_{n-m}^{*(l)},
$$

where $b_{n}^{*(l)}$ are the order l type 2 Bernoulli polynomials of the second kind.

Proof By replacing $t$ with $2 \log (1+t)$ in (23), from (11) and (17), the left-hand side of (23) is

$$
\begin{aligned}
1+J_{k}\left(x\left((1+t)-(1+t)^{-1}\right)\right. & =1+\sum_{l=1}^{\infty} \frac{x^{l}\left((1+t)-(1+t)^{-1}\right)^{l}}{(l-1) ! l^{k}} \\
& =1+\sum_{l=1}^{\infty} \frac{2^{l} x^{l}}{l^{k-1}}\left(\frac{(1+t)-(1+t)^{-1}}{2 \log (1+t)}\right)^{l} \frac{\log (1+t)^{l}}{l !} \\
& =1+\sum_{l=1}^{\infty} \frac{2^{l} x^{l}}{l^{k-1}} S_{1}(m, l) \frac{t^{m}}{m !} \sum_{j=0}^{\infty} b_{j}^{*(l)} \frac{t^{j}}{j !} \\
& =1+\sum_{m=1}^{\infty} \sum_{l=1}^{m} \frac{2^{l} x^{l}}{l^{k-1}} S_{1}(m, l) \frac{t^{m}}{m !} \sum_{j=0}^{\infty} b_{j}^{*(l)} \frac{t^{j}}{j !} \\
& =1+\sum_{n=1}^{\infty} \sum_{m=1}^{n} \sum_{l=1}^{m}\left(\begin{array}{c}
n \\
m
\end{array}\right) \frac{2^{l} x^{l}}{l^{k-1}} S_{1}(m, l) b_{n-m}^{*(l)} \frac{t^{n}}{n !} .
\end{aligned}
$$

On the other hand, by (11), the right-hand side of (23) is

$$
\begin{aligned}
\sum_{m=0}^{\infty} B_{m}^{(c, k)}(x) \frac{(2 \log (1+t))^{m}}{m !} & =\sum_{m=0}^{\infty} 2^{m} B_{m}^{(c, k)}(x) \sum_{n=m}^{\infty} S_{1}(n, m) \frac{t^{n}}{n !} \\
& =\sum_{n=0}^{\infty} \sum_{m=0}^{n} 2^{m} S_{1}(n, m) B_{m}^{(c, k)}(x) \frac{t^{n}}{n !} \\
& =1+\sum_{n=1}^{\infty} \sum_{m=0}^{n} 2^{m} S_{1}(n, m) B_{m}^{(c, k)}(x) .
\end{aligned}
$$

Since $S_{1}(n, 0)=0$ for $n \geq 1$, by comparing with the coefficients of (31) and (32), we have the desired result.

Theorem 7 For $k \in \mathbb{Z}$ and $n \geq 1$, we have

$$
\sum_{j=0}^{n}\left(\begin{array}{l}
n \\
j
\end{array}\right) b_{j} B_{n-j+1}^{(c, k)}(x)=\sum_{m=0}^{n}\left(\begin{array}{c}
n \\
m
\end{array}\right) E_{n-m} B_{m+1}^{(c, k)}(x),
$$

where $b_{n}$ are the ordinary Bernoulli numbers and $E_{n}$ are the ordinary Euler numbers. 
Proof Differentiating with respect to $t$ in (23), the left-hand side of (23) is

$$
\begin{aligned}
\frac{\partial}{\partial t}\left(1+J_{k}\left(x\left(e^{\frac{t}{2}}-e^{-\frac{t}{2}}\right)\right)\right) & =\sum_{n=1}^{\infty} \frac{x^{n}\left(e^{\frac{t}{2}}-e^{-\frac{t}{2}}\right)^{n-1}}{(n-1) ! n^{k-1}} \frac{1}{2}\left(e^{\frac{t}{2}}+e^{-\frac{t}{2}}\right) \\
& =\frac{e^{\frac{t}{2}}+e^{-\frac{t}{2}}}{2\left(e^{\frac{t}{2}}-e^{-\frac{t}{2}}\right)} \sum_{n=1}^{\infty} \frac{x^{n}\left(e^{\frac{t}{2}}-e^{-\frac{t}{2}}\right)^{n}}{(n-1) ! n^{k-1}} \\
& =\frac{e^{\frac{t}{2}}+e^{-\frac{t}{2}}}{2\left(e^{\frac{t}{2}}-e^{-\frac{t}{2}}\right)} J_{k-1}\left(x\left(e^{\frac{t}{2}}-e^{-\frac{t}{2}}\right)\right) \\
& =\frac{e^{t}+1}{2\left(e^{t}-1\right)} \sum_{n=1}^{\infty} B_{n}^{(c, k)}(x) \frac{t^{n}}{n !}
\end{aligned}
$$

On the other hand, the right-hand side of (23) is

$$
\frac{\partial}{\partial t}\left(\sum_{n=0}^{\infty} B_{n}^{(c, k)}(x) \frac{t^{n}}{n !}\right)=\sum_{n=0}^{\infty} B_{n+1}^{(c, k)}(x) \frac{t^{n}}{n !}
$$

Combining (33) with (34), we get

$$
\frac{1}{e^{t}-1} \sum_{n=1}^{\infty} B_{n}^{(c, k)}(x) \frac{t^{n}}{n !}=\frac{2}{e^{t}+1} \sum_{n=0}^{\infty} B_{n+1}^{(c, k)}(x) \frac{t^{n}}{n !}
$$

From (16) and (35), we have

$$
\sum_{j=0}^{\infty} b_{j} \frac{t^{j}}{j !} \sum_{m=0}^{\infty} \frac{1}{m+1} B_{m+1}^{(c, k)}(x) \frac{t^{m}}{m !}=\sum_{i=0}^{\infty} E_{i} \frac{t^{i}}{i !} \sum_{m=0}^{\infty} B_{m+1}^{(c, k)}(x) \frac{t^{m}}{m !}
$$

By (36), we have

$$
\sum_{n=0}^{\infty} \sum_{j=0}^{n}\left(\begin{array}{l}
n \\
j
\end{array}\right) b_{j} B_{n-j+1}^{(c, k)}(x) \frac{t^{n}}{n !}=\sum_{n=0}^{\infty} \sum_{m=0}^{n}\left(\begin{array}{l}
n \\
m
\end{array}\right) E_{n-m} B_{m+1}^{(c, k)}(x) \frac{t^{n}}{n !}
$$

By comparing with the coefficients of both sides of (37), we have the desired result.

\section{Further remark}

Let $r \in \mathbb{N} \cup\{0\}$, the $r$-Stirling numbers $S_{2, r}(n, j)$ of the second kind are given by

$$
\frac{1}{j !} e^{r t}\left(e^{t}-1\right)^{j}=\sum_{n=j}^{\infty} S_{2, r}(n+r, j+r) \frac{t^{n}}{n !} \quad(\text { see }[15]) .
$$

In view of (14), the $r$-Bell polynomials are given by

$$
\operatorname{bel}_{n}^{(r)}(x)=\sum_{j=0}^{n} S_{2, r}(n+r, j+r) x^{j} \quad(n \geq 0),(\text { see }[15]) .
$$


From (38), it is easy to show that the generating function of degenerate $r$-Bell polynomials is given by

$$
e^{r t} e^{x\left(e_{\lambda}(t)-1\right)}=\sum_{n=0}^{\infty} b e l_{n, r}(x \mid \lambda) \frac{t^{n}}{n !} \quad(\text { see }[14])
$$

When $x=1$, $b e l_{n}^{(r)}(\lambda)=b e l_{n}^{(r)}(1 \mid \lambda)$ which are called the degenerate $r$-Bell numbers.

We can define the extended poly-Bell polynomials $\mathfrak{B e r}_{n, \lambda}^{(k)}(x)$, which are derived from the polyexponential function to be

$$
1+J_{k}\left(x\left(e^{t}-1\right)+r t\right)=\sum_{n=0}^{\infty} \mathfrak{B e l}_{n}^{(k)}(x) \frac{t^{n}}{n !} \quad \text { and } \quad \mathfrak{B e l}_{0}^{(k)}(x)=1
$$

When $x=1, \mathfrak{B e l}_{n}^{(k)}=\mathfrak{B e l}_{n}^{(k)}(1)$ which are called the extended poly-Bell numbers.

When $k=1$, we note that

$$
1+J_{k}\left(x\left(e^{t}-1\right)+r t\right)=1+\sum_{n=1}^{\infty} \frac{\left(x\left(e^{t}-1\right)+r t\right)^{n}}{n !}=e^{x\left(e^{t}-1\right)+r t}=e^{x\left(e^{t}-1\right)} e^{r t}=b e l_{n, r}(x) .
$$

In particular, when $r=-\frac{1}{2}, \mathfrak{B e l}_{n,-\frac{1}{2}}(x)=B_{n}^{(c)}(x)$.

Theorem 8 For $n \geq 1$, we have

$$
\mathfrak{B e l}_{n, r}^{(k)}(x)=\sum_{l=0}^{m}\left(\begin{array}{c}
n \\
m-l
\end{array}\right) \frac{r^{m-l}}{m^{k-1}} S_{2}(n+l-m, l)
$$

Proof From (13) and (41), we observe that

$$
\begin{aligned}
& 1+J_{k}\left(x\left(e^{t}-1\right)+r t\right)=1+\sum_{m=1}^{\infty} \frac{\left(x\left(e^{t}-1\right)+r t\right)^{m}}{(m-1) ! m^{k}} \\
&=1+\sum_{m=1}^{\infty} \frac{1}{m^{k-1} m !} \sum_{l=0}^{m}\left(\begin{array}{c}
m \\
l
\end{array}\right) x^{l}\left(e^{t}-1\right)^{l}(r t)^{m-l} \\
&=1+\sum_{m=1}^{\infty} \sum_{l=0}^{m} \frac{r^{m-l} x^{l}}{m^{k-1}(m-l) !} t^{m-l} \frac{\left(e^{t}-1\right)^{l}}{l !} \\
&=1+\sum_{m=1}^{\infty} \sum_{l=0}^{m} \frac{r^{m-l} x^{l}}{m^{k-1}(m-l) !} \sum_{n=l}^{\infty} S_{2}(n, l) \frac{t^{n+m-l}}{n !} \\
&=1+\sum_{m=1}^{\infty} \sum_{l=0}^{m} \frac{r^{m-l} x^{l}}{m^{k-1}(m-l) !} \sum_{n=0}^{\infty} S_{2}(n+l, l) \frac{t^{n+m}}{(n+l) !} \\
&=1+\sum_{m=1}^{\infty} \sum_{l=0}^{m} \frac{r^{m-l} x^{l}}{m^{k-1}(m-l) !} \sum_{n=m}^{\infty} S_{2}(n+l-m, l) \frac{t^{n}}{(n+l-m) !} \\
&=1+\sum_{n=1}^{\infty} \sum_{m=1}^{n} \sum_{l=0}^{m}\left(\begin{array}{c}
n-l) \\
m^{m-l}
\end{array}\right. \\
& m^{k-1} S_{2}(n+l-m, l) \frac{t^{n}}{n !}
\end{aligned}
$$

By (41) and (42), we get what we want. 


\section{Conclusion}

To summarize, we introduced poly-central factorial sequences and poly-central Bell polynomials in terms of the polyexponential functions, reducing them to central factorials and central Bell polynomials of the second kind respectively when $k=1$. We derived relations between poly-central factorial sequences and power of $x$ in Theorems 1, 2. We also obtained relations between poly-central Bell polynomials and power of $x$ in Theorems 3, 4 . In addition, we showed several identities between poly-central Bell polynomials and polyBell polynomials; between poly-central Bell polynomials and higher order type 2 Bernoulli polynomials of second kind; recurrence formula of poly-central Bell polynomials in Theorems $5,6,7$.

To conclude, there are various methods for studying special polynomials and numbers, including: generating functions, combinatorial methods, umbral calculus, differential equations, and probability theory $[2,4-7,18-20]$. We are now interested in continuing our research into the application of 'poly' versions of certain special polynomials and numbers in the fields of physics, science, and engineering as well as mathematics.

\section{Acknowledgements}

The authors would like to thank Jangjeon Institute for Mathematical Science for the support of this research.

\section{Funding}

This work was supported by the Basic Science Research Program, the National Research Foundation of Korea (NRF-2021R1F1A1050151).

\section{Availability of data and materials}

This paper does not use data and materials.

\section{Declarations}

Ethics approval and consent to participate

The authors reveal that there is no ethical problem in the production of this paper.

Consent for publication

The authors want to publish this paper in this journal.

Competing interests

The authors declare that they have no competing interests.

\section{Authors' contributions}

TK and HKK conceived the framework and structured the whole paper; HKK wrote the whole paper. TK and HKK checked the results of the paper and completed the revision of the article. All authors read and approved the final manuscript.

\section{Author details}

'Department of Mathematics Education, Daegu Catholic University, Gyeongsan 38430, Republic of Korea. ${ }^{2}$ Department of Mathematics, Kwangwoon University, Seoul 139-701, Republic of Korea.

\section{Publisher's Note}

Springer Nature remains neutral with regard to jurisdictional claims in published maps and institutional affiliations.

Received: 27 August 2021 Accepted: 9 November 2021 Published online: 22 November 2021

\section{References}

1. Bayad, A., Hamahata, Y.: Polylogarithms and poly-Bernoulli polynomials. Kyushu J. Math. 65(1), 15-24 (2012)

2. Butzer, P.L., Schmidt, M., Stark, E.L., Vogt, L.: Central factorial numbers; their main properties and some applications. Numer. Funct. Anal. Optim. 10(5-6), 419-488 (1989)

3. Carlitz, L.: Degenerate Stirling, Bernoulli and Eulerian numbers. Util. Math. 15, 51-88 (1979)

4. Charalambides, C.A.: Central factorial numbers and related expansions. Fibonacci Q. 19, 451-456 (1981)

5. Comtet, L: Advanced Combinatorics: The Art of Finite and Infinite Expansions. Reidel, Dordrecht (1974)

6. Eastwood, M., Goldschmidt, H.: Zero-energy fields on complex projective space. J. Differ. Geom. 94(1), 1-186 (2013)

7. Everitt, W.N., Kwon, K.H., Littlejohn, L.L., Wellman, R., Yoon, G.J.: Jacobi-Stirling numbers, Jacobi polynomials, and the left-definite analysis of the classical Jacobi differential expression. J. Comput. Appl. Math. 208, 29-56 (2007) 
8. Hardy, G.H.: On a class analytic functions. Proc. Lond. Math. Soc. (2) 3(1), 441-460 (1905)

9. Kim, D.S., Kim, T.: A note on polyexponential and unipoly functions. Russ. J. Math. Phys. 26(1), 40-49 (2019)

10. Kim, T.: A note on central factorial numbers. Proc. Jangjeon Math. Soc. 21, 575-588 (2018)

11. Kim, T., Kim, D.S.: A note on central Bell numbers and polynomials. Russ. J. Math. Phys. 27(1), 76-81 (2020)

12. Kim, T., Kim, D.S., Dolgy, D.V., Lee, S.-H., Kwon, J.: Some identities of the higher-order type 2 Bernoulli numbers and polynomials of the second kind. Comput. Model. Eng. Sci. 128(3), 1121-1132 (2021)

13. Kim, T., Kim, D.S., Kim, H.Y., Kwon, J.: Some results on degenerate Daehee and Bernoulli numbers and polynomials. Adv. Differ. Equ. 2020, 311 (2020)

14. Kim, T., Kim, D.S., Kwon, J.K., Lee, H.S.: Degenerate polyexponential functions and type 2 degenerate poly-Bernoulli numbers and polynomials. Adv. Differ. Equ. 2020, 168 (2020)

15. Kim, T., Kim, D.S., Lee, H., Park, J.-W.: A note on degenerate $r$-Stirling numbers. J. Inequal. Appl. 2020,225 (2020)

16. Kim, T., Kim, H.K.: Degenerate poly-Bell polynomials and numbers. Adv. Differ. Equ. 2021, 361 (2021)

17. Kim, T., Kim, H.K.: Degenerate poly-Lah Bell polynomials and numbers. https://doi.org/10.13140/RG.2.2.19672.83208

18. Loureiro, A.F.: New results on the Bochner condition about classical orthogonal polynomials. J. Math. Anal. Appl. 364, 307-323 (2010)

19. Riordan, J.: Combinatorial Identities. Wiley, New York (1968)

20. Shadrin, S., Spitz, L., Zvonkine, D.: On double Hurwitz numbers with completed cycles. J. Lond. Math. Soc. 86(2), 407-432 (2012)

\section{Submit your manuscript to a SpringerOpen ${ }^{\circ}$ journal and benefit from:}

- Convenient online submission

- Rigorous peer review

- Open access: articles freely available online

- High visibility within the field

- Retaining the copyright to your article

Submit your next manuscript at $\gg$ springeropen.com 